\title{
Shakespeare und England im 16. Jahrhundert ${ }^{1}$
}

\author{
Arnd Reitemeier (Universität Göttingen)
}

This royal throne of kings, this sceptred isle,

This earth of majesty, this seat of Mars,

This other Eden, demi-paradise,

This fortress built by Nature for herself

Against infection and the hand of war,

This happy breed of men, this little world,

This precious stone set in the silver sea,

Which serves it in the office of a wall,

Or as a moat defensive to a house,

Against the envy of less happier lands;

This blessed plot, this earth, this realm, this England,

This nurse, this teeming womb of royal kings,

Feared by their breed, and famous by their birth,

Renowned for their deeds as far from home,

For Christian service and true chivalry,

As is the sepulchre in stubborn Jewry

${ }^{1}$ Der folgende Beitrag ist eine überarbeitete und gekürzte Fassung des am 30.10.2014 gehaltenen Vortrags. Im Vortrag wurde ausführlich auf den Film „Elisabeth, The Golden Age“ (2007), Regie Shekhar Kapur, Bezug genommen, doch wurden die entsprechenden Passagen hier entfernt, da sie ohne Kenntnis des Films unverständlich sind. Anstelle von ausführlichen Literaturangaben wurde, mit Ausnahme von Zitaten, Zitatbelegen abgesehen, auf Einzelbelege verzichtet. 
Of the world's ransom, blessed Mary's son.

This land of such dear souls, this dear dear land,

Dear for her reputation through the world, Is now leased out $-\mathrm{I}$ die pronouncing it Like to a tenement or pelting farm.

Dieses Zitat aus Shakespeares Richard II. gehört zu den berühmtesten Sätzen der Weltliteratur und brachte in Teilen das Selbstbild Englands am Ende des 16. Jahrhunderts zum Ausdruck. Shakespeare thematisierte die Insellage Großbritanniens, die seiner Meinung nach besondere Beziehung zwischen Herrschern und Beherrschten sowie das Verhältnis Englands zur den Königreichen Europas und der übrigen Welt. Inhaltlich stellt Shakespeare eine Paradoxie in das Zentrum, die nachfolgend im Drama aufgelöst wird, nämlich dass das große und reiche England als kleine und armselige Macht wahrgenommen wird. Laut Shakespeare unterscheidet sich England aber von allen anderen Fürstentümern und Königreichen durch die die Einheit von Geschichte, Königtum, Bevölkerung und Christentum. Indem alle Elemente aufeinander bezogen werden, schreibt Shakespeare England gleichsam Modellcharakter als Königreich par excellence zu. Dieses Image wirkt in vielem bis heute sowohl in England als auch in der übrigen Welt fort, denn weiterhin wird englischen Institutionen Vorbildcharakter zugwiesen wie auch das gesellschaftliche Miteinander als Grundlage außenpolitischer Stärke beschrieben wird. Um beides zu erläutern gilt es die politischen Rahmenbedingungen zu analysieren:

Die englische Politik des 16. Jahrhunderts wurde von persönlichen Ambitionen bestimmt, die unmittelbar den Interessen der Dynastie verknüpft waren. Die sogenannten Rosenkriege als Konflikt zwischen verschiedenen Häusern desselben Herrscherhauses markierten in Vielem das Ende der politischen Ordnung Englands im Mittelalter. Nachdem zwei der Linien ausgestorben waren, konnte Heinrich VII. vorübergehend einen innenpolitischen Frieden erzielen und in Manchem Ausgleich schaffen. Weiterhin aber stritt eine Reihe bedeutender Adelsfamilien um Einfluss am Hof sowie um den Besitz von Grafschaften, Rechten und ökonomischen Ressourcen. Diese Auseinandersetzungen konnten auch unter Heinrich VIII. jederzeit wieder zu innenpolitischen Auseinandersetzungen führen, sobald nämlich die Führung und der Führungsanspruch der Dynastie in Frage gestellt wurden. Folglich bemühten sich die Herrscher seit Heinrich VII. um eine möglichst große Distanz zum Adel, und zum anderen galt das primäre Streben der englischen Herrscher während der ersten Hälfte des 16. Jahrhunderts der Sicherung des Fortbestands der Dynastie. 
Maßgeblich eben aus dieser Sorge suchte Heinrich VIII. nach der Möglichkeit der Scheidung von Katharina von Aragon. Diese war kirchenrechtlich gemäß spezifischer Bedingungen nicht ausgeschlossen, allerdings verweigerte Katharina ihre Zustimmung. Zugleich übte ihr Neffe, Kaiser Karl V., nach der Eroberung Roms im Jahr 1527 dominierenden Einfluss auf den Papst aus und stand damit der gewünschten Ehetrennung ablehnend gegenüber. Beraten durch Thomas Cranmer und Thomas Cromwell initiierte Heinrich VIII. eine in dieser Form nie dagewesene Gesetzgebung, konnte er doch das englische Parlament davon überzeugen, dass er nur Gott gegenüber verantwortlich sei. Hintergrund hierfür war maßgeblich das Mitspracherecht des englischen König bei der Einsetzung von Bischöfen und Äbten, das die englischen Herrscher in Folge des Investiturstreits erlangt hatten. Dieses war bereits im 14. und 15. Jahrhundert zur Einschränkung der päpstlichen Rechte in England genutzt worden, so dass es für das englische Parlament gute Gründe gab, im Jahr 1534 den Act of Supremacy zu beschließen, wonach „the king shall be taken, accepted and reputed the only supreme head in earth of the Church of England called Anglicana Ecclesia“. Indem der König nun an der Spitze der Church of England stand, konnte die Ehe auch des Herrschers vor einem weltlichen Gericht - und damit ohne Hinzuziehung des Papstes - geschieden werden. Zweifelsfrei aber hielt Heinrich VIII. persönlich am katholischen Glauben fest, während es in der englischen Bevölkerung erhebliche Kritik am Klerus und besonders an der römischen Kurie gab, was der Akzeptanz lutherischer Ideen den Boden bereitete.

Die Schaffung einer mit Territorium und Herrscher verknüpften kirchlichen Einheit wurde auch in anderen Territorien in Europa angestrebt, doch langfristig entscheidend wurde die administrativ-politische ebenso wie religiöse Ausgestaltung der Church of England. Nach und nach wurden protestantische Inhalte eingeführt, denn bereits unter Heinrich VIII. wurden die sogenannten Ten Articles erlassen, die die Heilige Schrift als Norm definierten und die Sakramente auf Taufe, Buße und Abendmahl beschränkten. Hinzu trat die volksprachliche Bibelübersetzung von Tyndale, die unter Edward VI. mit dem Book of Common Prayer als Agende der Church of England ergänzt wurde, welche sich allerdings in Vielem noch am alten Glauben orientierte. Maria trat als Königin für eine Rekatholisierung ein, doch weite Teile der englischen Bevölkerung wehrten sich gegen die Verfolgung von Protestanten als Ketzern. Nach ihrem Amtsantritt bestätigte Elisabeth die Agende. Diese trat in den Jahrzehnten ihrer Herrschaft für die Durchsetzung des christlichen Glaubens ein und verfolgte keine konfessionelle Leitidee jenseits der Church of England und der hiermit verbundenen Ablehnung des Katholizismus. Damit öffnete sie der Entwicklung und Ausbreitung des Puritanismus breiten 
Raum, so dass sich besonders in Schottland sowie im Norden Englands ein immer größer werdender Teil der Bevölkerung dieser Ausprägung des Calvinismus zuwandte. Letztlich war die englische Bevölkerung am Ende des 16. Jahrhunderts zerrissen, auch wenn sie in ihrer Mehrheit der katholischen Kirche fern stand. Dynastisch gesehen konnte Maria Stuart während der ersten Jahrzehnte der Herrschaft Elisabeths als Alternative gesehen werden, die aber wegen ihres katholischen Glaubens und ihrer französischen Erziehung Unterstützung durch europäische Dynastien von weiten Teilen nicht nur des englischen Adels abgelehnt wurde. Unter Elisabeth wurde die „ecclesia Anglicana“ im Inneren weiter ausgebaut, doch die Herrscherin verfolgte - anders als ihre beiden Vorgänger kein konfessionelles Programm sondern beschränkte sich auf Versuche, den christlichen Glauben tiefer als zuvor zu verankern.

Die innenpolitischen Schwierigkeiten wurden durch außenpolitische Unsicherheiten verschärft. In Folge der Trennung und nachfolgenden Scheidung Heinrichs VIII. von Katharina von Aragon im Jahr 1533 verschlechterte sich das englisch-spanische Verhältnis. Die drohende außenpolitische Isolierung konnte jedoch vermieden werden, da sich das Verhältnis Englands zu Frankreich entspannte, auch weil sich in Frankreich die innenpolitischen und konfessionellen Schwierigkeiten verschärften. Aus dem Bestreben des englischen Königs nach einer Ehetrennung entwickelte sich jedoch eine konfessionelle Auseinandersetzung, die wesentliche Teile Europas ergriff, denn ungewollt eröffnete die Politik Heinrichs VIII. den ebenso altgläubigen wie miteinander verwandten Monarchen Spaniens und des Reichs, gegen England vorzugehen. Unterstützt von der Kurie wurde der sich bis in das 17. Jahrhundert hinziehende Krieg Spaniens gegen England als Kampf gegen den Protestantismus geführt. Um die hieraus sich ergebende teilweise Isolation Englands aufzuheben war es strategisch wie dynastisch nachvollziehbar, dass die katholische Tochter Heinrichs VIII., Maria, 1554 den spanischen Thronfolger Philipp I. heiratete und auf diese Weise den Krieg vorübergehend beendete. Im Zuge der Heiratsverhandlungen aber konnte der englische Adel durchsetzen, dass dem spanischen Thronfolger jede Form der Herrschaftsberechtigung verweigert wurde, so dass dieser nach dem Tod Marias im Jahr 1588 England wieder verlassen musste. Die dritte Tochter Heinrichs VIII., Elisabeth, suchte nun die Wiederaufnahme des Kriegs zu vermeiden und verhandelte lange über eine Heirat mit Philipp II. Hierzu aber kam es aus einer komplexen Mischung politischer, religiöser und persönlicher Gründe nicht, was Spanien als Düpierung ansah. Der nun wieder aufflammende Krieg zog sich von 1585 bis 1604 hin und ergriff ebenso die Niederlande, Frankreich wie Irland. In der allgemeinen Wahrnehmung in beiden Ländern wie in Europa bildete der mit der Great Armada 
von 1588 verknüpfte spanische Landungsversuch den Höhepunkt der Auseinandersetzungen.

Hintergrund für den während der Herrschaft Elisabeths virulenten spanischenglischen Konflikt war zum einen die Trennung zwischen der österreichischen und der spanischen Linie der Habsburger, so dass sich die Auseinandersetzungen zwischen den habsburgischen Monarchen und den französischen Königen um die Vorherrschaft im westlichen Mittelmeer beruhigten. Dies ermöglichte es Frankreich im Jahr 1558 die Stadt und Festung Calais als letzten Festlandbesitz Englands zu erobern. Sechs Jahre später - im Geburtsjahr Shakespeares - verzichtete Elisabeth gegen Zahlung einer umfangreichen Geldsumme auf alle Ansprüche auf Calais, da England eine Fortsetzung der Auseinandersetzungen auf dem Kontinent nicht länger finanzieren konnte. Zeitlich nahezu parallel wurde auf dem Augsburger Reichstag von 1555 mit der Formel „cuius regio eius religio“ ein vorübergehender Modus vivendi zwischen den katholischen und den lutherischen Reichsständen gefunden, so dass sich die Gesamtsituation in Europa vorübergehend beruhigte. In Frankreich starben nach dem Tod Heinrichs II. im Jahr 1559 kurz nacheinander seine drei Söhne. Zum einen kehrte sodann die Ehefrau von Franz II., Maria Stuart, im Jahr 1561 nach Schottland zurück und vertrat nun ihre Erbansprüche auf den englischen Thron. Zum anderen traten nun in Frankreich, aber auch in Schottland, politisch-soziale ebenso wie religiöse Spannungen offen zutage, denn in beiden Ländern wandten sich Teile des Adels gegen die jeweils herrschende Dynastie, und in beiden Ländern kam es zu wachsenden Auseinandersetzungen zwischen Katholiken, Protestanten und Calvinisten. Einerseits boten diese inneren Auseinandersetzungen der englischen Königin die Möglichkeit der indirekten Einflussnahme. Doch drohte England immer wieder in die Feindseligkeiten hineingezogen werden - besonders nachdem Maria Stuart nach England geflohen war und nach einem vermeintlichen Komplott gegen Elisabeth hingerichtet wurde, was Spanien einen zusätzlichen Grund zum Vorgehen gegen England lieferte.

Indem, wie oben dargelegt, die Notwendigkeit der Distanzierung der englischen Herrscher besonders von den mächtigen Adelsdynastien innenpolitische Konsequenz der Rosenkriege war, kam der Heiratsverbindung des Herrschers bzw. des Thronprinzen politisch wie sozial zentrale Bedeutung zu, da mit dieser der Aufstieg oder Fall einzelner Familien verbunden war. Einer der Gründe für die Ehelosigkeit Elisabeths I. war daher das Fehlen einer sozial wie politisch adäquaten Partie in Europa. Doch umgekehrt eröffnete der Zugang zum Hof die bedeutendste Möglichkeit der Gunstbezeugung für die Mitglieder des Adels, denn einzig die Krone konnte Standesveränderungen vornehmen und Staatsämter verleihen. 
Heinrich VIII. wie auch teilweise Elisabeth I. gelang es in überaus komplizierten wie komplexen Prozessen, einzelnen Personen und Familien eine vorübergehende Möglichkeit der Partizipation an der Macht einzuräumen. Dies wurde durch überaus differenzierte Symbolik am Hof verstärkt mit der Nebenfolge, dass nicht nur die Hofhaltung erhebliche Summen verschlang, sondern dass der Adel diese ebenso aufwändige wie kostspielige Form sozialer und politischer Differenzierung mittragen musste. Im Ergebnis wurde ein insgesamt politisch weitgehend stabiles System, das auf den Herrscher zugeschnitten war, wiederholt in einen labilen Zustand versetzt mit dem Erfolg, dass die Macht des Herrschers wuchs und seine Stellung unangefochten war. Ergänzend stützten sich Heinrich VIII., verstärkt aber noch Elisabeth I. auf den niederen Adel, aus deren Kreis beispielsweise die justices of the peace berufen wurden, die auf den Herrscher ad personam einen Eid ablegen mussten und deren Aufgabe die Durchsetzung der zentral verfügten Rechts war. Mit dieser Förderung schufen die Herrscher ein Netzwerk loyaler Machtträger, deren sozialer Aufstieg in ihrer Hand lag.

Die innenpolitischen Auseinandersetzungen des ausgehenden 15. Jahrhunderts, die Kriege gegen Frankreich und Spanien sowie die Hofhaltung verschlangen enorme Summen, so dass alle englischen Monarchen stets zusätzliche Einnahmequellen suchten. Wie auch anderswo in Europa verknüpften die Herrscher daher die Kritik am Klerus mit dem Zugriff auf die Einnahmen der Kirche. Nach einer Visitation der Klöster unter Thomas Cromwell wurden auf Beschluss des Parlaments zunächst die kleinen Klöster, dann im Jahr nahezu sämtlichen Klöster aufgelöst. Während die Kleriker mit Pensionen abgefunden wurden, fielen der Grundbesitz und sämtliche an die Krone. Heinrich VIII. nutzte dies zur Stärkung seiner Position, denn nicht nur wurde der Klerus deutlich reduziert und geschwächt, sondern der Adel wurde zusätzlich an die Krone gebunden, da diese den enteigneten Grundbesitz der Kirche zur eigenen Besitzarrondierung erwarb. Auch aus diesem Grund stießen die Bemühungen Marias I. um eine Rekatholisierung auf erhebliche Widerstände, denn der Adel fürchtete eine Rückgängigmachung dieser Grundbesitzübertragungen. Umgekehrt sorgte Elisabeths Festhalten am Status quo für innenpolitische Stabilität.

Dieser politischen Stabilität im Inneren standen wesentliche ökonomische Umwälzungen gegenüber, da die englische Ökonomie war zu Beginn des 16. Jahrhunderts in Europa von lediglich geringer Bedeutung war. Lediglich die Niederlande bezogen in nennenswertem Maß Rohwolle aus England, doch in Folge sowohl der wachsenden wirtschaftlichen Bedeutung der Niederlande als auch der konfessionellen wie politischen Auseinandersetzungen in den Niederlanden geriet dieser Absatz in Gefahr. Heinrich VIII. und Maria I. versuchten daher eine 
Tuchindustrie in England aufzubauen, um die Abhängigkeit von den Wollexporten zu verringern und um die Gewinnspannen durch die Ausschaltung des Zwischenhandels zu steigern. Nachfolgend begann das Zeitalter der „enclosures“, denn besonders Großgrundbesitzer versuchten die Allmenden zur effektiven Bewirtschaftung entweder zu verpachten oder selbst zu nutzen. Ergänzend wurden die königlichen Wälder, die um die Klosterforsten vermehrt worden waren, verpachtet und zunehmend abgeholzt, denn nicht nur erbrachte die Schafhaltung substantielle Gewinne, sondern das Holz wurde von den Werften für den Bau von Schiffen abgenommen. Die Herrscher förderten die Wirtschaft, indem importierte Güter höher besteuert wurden als exportierte. Auch wurde die Zollverwaltung reformiert, um höhere Einnahmen zu erzielen. Viele der Maßnahmen erbrachten der Krone kurzfristige Einnahmen, zeigten aber nur langfristig Wirkung. Die Bedeutung des englischen Überseehandels war zu Beginn des 16. Jahrhunderts eher gering und wuchs auch bis zum Beginn des 17. Jahrhunderts nur langsam. England partizipierte zunächst nur marginal am Handel über den Atlantik. In der zweiten Hälfte des 16. Jahrhunderts versuchten einzelne Kaufleute den spanischen und portugiesischen Seefahrern nachzueifern. Gewinne wie die von Walter Raleigh erzielten nur wenige. Da es gleichzeitig in der zweiten Hälfte des Jahrhunderts die Inflation in England wie überall in Europa deutlich zunahm, war es um die englischen Staatsfinanzen nicht gut bestellt. Die Friedensanstrengungen Elisabeths basierten daher ganz wesentlich auf ökonomischen Zwängen.

Folge der wirtschaftlichen Schwierigkeiten war jedoch kein Anwachsen der Macht des Parlaments. Im Vergleich zum späten Mittelalter hatte dieses im Zeitalter der Rosenkriege wesentlich an Bedeutung verloren. Heinrich VIII. achtete zwar die Rechte des Parlaments, aber Thomas Cromwell gelang es lange Zeit, klare Mehrheiten für die Politik der Krone zu organisieren. Unter Elisabeth I. kam es zu keiner grundlegenden Änderung, denn diese berief während ihrer 45-jährigen Herrschaft lediglich zehn Parlamente ein, die ihren Vorschlägen und Bitten um die Gewährung von Steuern insbesondere wegen der andauernden außenpolitischen Bedrohung folgten.

An das eingangs angeführte Zitat anknüpfend verbrachte Shakespeare also seine Jugend und sein Leben in einem konfessionell zerrissenen Land, dessen Dynastie von allen wesentlichen englischen Akteuren als alternativlos betrachtet wurde und die folglich unangefochten an der Spitze stand, die das Land jedoch in Erbauseinandersetzungen verstrickte. Eine Gruppe adliger Familie teilte alle wesentlichen Ämter, Posten und Macht unter sich auf, sorgte sich aber weniger um das Land als vielmehr um die eigene Macht. Die Wirtschaft durchlief mühsam einen tiefgreifenden und langandauernden Wandlungsprozess. Der Krone fehlte es 
an Einnahmen und sie hatte Mühe auch nur das Militär zu finanzieren. England hatte sämtlichen Kontinentalbesitz verloren, befand sich militärisch in der Defensive und lebte fast eine Generation lang in der Gefahr einer Invasion. Und an der Spitze des Königreiches stand eine unverheiratete Frau, die sich aus einer Vielfalt an Gründen nicht zu einer Heirat mit einem der vielen Kandidaten aus dem englischen oder europäischen Hochadel entschließen konnte, die mehrfach Attentate der katholischen Opposition nur knapp überlebte und der es nur mit Mühe gelang, das Land in Anbetracht äußerer Gefahr zusammenzuhalten.

Hinzu kam: Die zweite Hälfte des 16. Jahrhunderts war eine Zeit der Wetterextreme, die manchmal, wie im Fall des Sturmes, der die spanische Flotte 1588 zerstreute, aus englischer Sicht Vorteile nach sich zogen, die vielfach aber Leid für die Menschen brachten. In Folge suchten die Menschen nach Erklärungen, die sie sowohl im christlichen Glauben als auch in vermeintlich übernatürlichen Phänomenen, Hexen etc. fanden.

Vor dem Hintergrund der zahlreichen politischen, ökonomische, sozialen und religiösen Schwierigkeiten inszenierte Elisabeth I. ihre Herrschaft in sehr wesentlichem Maß und achtete daher auf ihr Image, wie nicht zuletzt die zentralen Portraits belegen, die stets allegorisch wie symbolisch zu begreifen sind. Eben weil diese ikonographischen Werke nur mit Hintergrundwissen zu verstehen sind, wurde die Virgin Queen zur Projektionsfläche diverser Inhalte und Phantasien, was sich mit Shakespeares Instrumentalisierung historischer Phänomene deckt.

Shakespeare zog in seinen Werken immer wieder Analogien zwischen der Vergangenheit und der Gegenwart. Folglich maß er der Geschichte eine didaktische Funktion zum Verständnis der Gegenwart zu. In besonderem Maß galt dies für die Historiendramen, in denen die englische Geschichte des späten Mittelalters und der beginnenden Neuzeit thematisiert wurde. Doch auch diverse Komödien und Tragödien griffen Elemente historischer Ereignisse auf. Während Shakespeare in den Historien der Geschichte gleichsam nur eine Hintergrundfunktion für die eigentlich im Zentrum stehenden Protagonisten und ihrer jeweiligen Entwicklung zuwies, rückte er in den Tragödien jeweils das Schicksal des betrachteten Landes in den Vordergrund. Vor dem Hintergrund der äußeren und inneren politischen Verhältnisse gegen Ende des 16. Jahrhunderts ist es deswegen wenig verwunderlich, dass Shakespeare neun seiner zehn Historien in den 1590er Jahren verfasste. Seine übergeordnete Intention war somit die politische Selbstvergewisserung des englischen Königreichs mit Hilfe nicht zuletzt der eigenen Geschichte. Dieses Konzept war alles andere als neu, wie beispielsweise das Vorwort der englischen Plutarch-Übersetzung von 1579 zeigt: 
And we may well percieve how greatly we be beholden unto it, if we do no more but consider in how horrible darkness, and in how beastly and pestilent a quagmire of ignorance we should be plunged, if the remembrance of all the things that have been done ... were utterly drowned and forgotten. ${ }^{2}$

Shakespeare trat in seinen Werke für ein vergleichsweise konservatives Weltbild ein, das nicht von dynamischen Entwicklungen sondern von einer festgefügten Ordnung geprägt wurde. Diese Ordnung wurde in seinen Werken jeweils zu Beginn gestört, jedoch am Ende wieder hergestellt. Die hiermit verknüpften Vorstellungen eines gottgewollten Aufbaus der Welt waren weit verbreitet. Allerdings kam es Shakespeare nicht auf historische Exaktheit an. Zwar legte er vielen seiner Dramen die Tudor Chronicles zugrunde, doch ihm ging es um religiöse, ästhetische, politische und gesellschaftliche Fragen und nicht um wissenschaftliche Genauigkeit. Folglich kam es zu inhaltlichen und zeitlichen Verdichtungen mit dem Ziel, die für Shakespeare wichtigen Mechanismen sichtbar zu machen. Folglich gibt es Verknüpfungen zwischen Shakespeare und seinem Werk und dem von Shakespeare erlebten England des 16. Jahrhunderts, doch sind diese Verknüpfungen einseitig und lassen folglich nur bedingt Aussagen über das Land und seine Gesellschaft zu.

\section{Bibliographie}

Aaron, Melissa D. Global economics: A history of the theatre business, the Chamberlain's Men/King's Men and their plays 1599-1642. Newark: 2005.

Archer, Ian W. Hg. English Historical Documents, Bd. 5a: 1558-1603. London: 2011.

Aronson, Alex. Psyche und Symbol in Shakespeare, Bern: 1951.

Asquit, Claire. Shadowplay, the hidden Beliefs and coded Politics of William Shakespeare. New York: 2005.

Baker, D. British identities and English Renaissance literature. Hg. W. Maley. Cambridge: 2002.

\footnotetext{
2 Thomas North (Hg.), Plutarch, The Lives of the Nobel Grecians and Romans, London 1579, S. 3 , Zitat nach: Hester Lees-Jeffries, Shakespeare and memory, Oxford 2013, S. 61.
} 
Barroll, Leeds. Politics, Plague and Shakespeare's Theatre: the Stuart Years. London: 1991.

Black, Jeremy. A History of the British Isles. New York: 1996.

Black, John Bennett. The Oxford History of England, Bd. 8: The reign of Elizabeth, 1558-1603. Oxford: 1936.

Bradshaw, Brendan Hg.. British consciousness and identity. The making of Britain 1533-1707. Cambridge: 1998.

Brower, Rueben. Hero \& Saint, Shakespeare and the Graeco-Roman heroic Tradition. Oxford: 1973.

Burrow, Colin. Shakespeare and classic Antiquity. Oxford: 2013.

Cahill, Patricia A. „Nation formation and the English history plays“, A Companion to Shakespeare's Works: the Histories. Hgs. Richard Dutton, Jean Howard, E. Oxford: 2006. 70-93.

Canny, Nicholas P. The Oxford History of the British Empire, Bd. 1: The origins of Empire. British overseas enterprise to the close of the seventeenth century. Oxford: 1998.

Clapp, Brian William Hg. Documents in English Economic History, Bd. 1: England from 1000 to 1760. London: 1984.

Desmet, Christy. Reading Shakespeares Characters. Amherst, 1992.

Driver, Tom Faw. The sense of History in Greek and Shakespearean Drama. New York: 1960.

Elton, Geoffrey Rudolph. England under the Tudors. London: 1991.

---. The new History of England, Bd. 2: Reform and Reformation. England 15091558. London: 1977.

Elton, Geoffrey Rudolph. Tudor Revolution in Government. Administrative changes in the reign of Henry VIII. London u.a.: 1974.

Fox, Alistair/Guy, John. Reassessing the Henrician Age. Humanism, Politics and Reform 1500-1550. Oxford: 1986.

Graves, Michael. Elisabethan Parliaments 1559-1601. London u.a.: 1987.

Graves, Michael. The Tudor Parliaments. Crown, Lords and Commons 1485-1603. London u.a.: 1986. 2. Auflage 
Greenblatt, Stephen. Verhandlungen mit Shakespeare, Innenansichten der englischen Renaissance. Berlin: 1990.

Guy, John. Tudor England. Oxford u.a.: 1988.

Haan, Heiner/Niedhart, Gottfried. Geschichte Englands vom 16. bis zum 18. Jahrhundert. München: 1993.

Hattaway, Michael. Elizabethan Popular Theatre. London: 1982.

Holderness, Graham. „What is my nation: Shakespeare and national identities“, Shakespeare's Histories. Hg. Emma Smith. Oxford: 2004. 225-245.

Hopkins, Lisa. Drama and the succession to the crown, 1561 - 1633. Farnham: 2011.

Innes, Michael. Character and Motive in Shakespeare. London: 1951.

Kirschbaum, Leo. Character and Characterization in Shakespeare. Heidelberg, 1975.

Lees-Jeffries, Hester. Shakespeare and Memory. Oxford, 2013.

MacGregor, Neil. Shakespeares ruhelose Welt. München: 2013.

Mackie, John Duncan. The Oxford History of England. Bd. 7: The Earlier Tudors, 1485-1558. Oxford: 1952.

Maitland, Frederic William. Constitutional History of England. Cambridge, 1968.

Maley, W. „'This sceptre isle': Shakespeare and the British problem“ Shakespeare and National Culture. Hg. J. J. Joughin. Manchester: 1997. 83-108.

Martindale, Charles: Raylor, A. B. Hg. Shakespeare and the Classics. Cambridge: 2004.

Martindale, Charles. Shakespeare and the Use of Antiquity, an Introductory. London: 1990.

Mathews, Honor. Character and Symbol in Shakespear's Plays. Cambridge, 1962.

Miola, Robert S. Shakespeares Rome. Cambridge, 1983.

Montrose, Louis. The Subject of Elizabeth: Authority, Gender and Representation. Chicago, 2006.

Moody, Theodore William Hg. u.a. A new History of Ireland, Bd. 3: Early Modern Ireland, 1534-1691. Oxford: 1991. 
Nuttall, Anthony D. A New Mimesis, Shakespeare and the Representation of Reality. London: 1983.

Orgel, Steven. Shakespeare and History. New York: 1999.

Pollard, Albert Frederick. The Evolution of Parliament. London: 1962. 2. Aufl.

Purdon, Noel. The Words of Mercury, Shakespeare and the English mythology of the Renaissance. Salzburg: 1974.

Purkiss, Diane. „Shakespeare, ghosts and popular folklore“, Shakespeare and Elizabethan popular culture. Hgs. Stuart Gillespie/Neil Rhodes. London: 2006. 136-154.

Rohrmoser, Günter. Shakespeare, Erfahrungen der Geschichte. München: 1971.

Schwanitz, Dietrich. Englische Kulturgeschichte. Tübingen: 1995. 2 Bde.

Sewell, Arthur. Character and Society in Shakespeare. Oxford, 1951.

Starkey, David. The Reign of Henry VIII. Personalities and Politics. London: 1985.

Stoll, Elmer Edgar. Art and Artifice in Shakespeare, a Study in Dramatic Contrast and Illusion. London: 1963.

Strier, Richard/Bevington, David Hgs. The theatrical city: culture, theatre and politics in London 1576-1649. Cambridge: 1995.

Thirsk, Joan. The Agrarian History of England and Wales, Bd. 4: 1500-1640.

Cambridge: 1967.

Walsh, Brian. Shakespeare, the Queen's Men and the Elizabethan Performance of History. Cambridge: 2009.

Wende, Peter. Das britische Empire. Geschichte eines Weltreiches. München: 2008.

Wende, Peter. Geschichte Englands. Stuttgart: 1995.

Wende, Peter. Großbritannien 1500 - 2000. München: 2001.

Wheale, Nigel. Writing and society: literacy, print and politics in Britain 1590-1660. London, New York: 1999.

Williams, Charles H. Hg.. English Historical Documents, Bd. 5: 1485-1558. London: 1967.

Williams, Glanmor. The History of Wales, Bd. 3: Recovery, Reorientation and Reformation. Wales c. 1514-1642. Oxford, 1987. 
Williams, Penry. The later Tudors. England 1547-1603. Oxford u.a.: 1995.

Wormald, Jenny u.a. Hg. The new History of Scotland, Bd. 4: Court, Kirk, and Community. Scotland 1470-1625. London: 1981. 